\title{
Totalidad y complejidad. Notas sobre la concepción del pensamiento complejo
}

\author{
Jorge Arturo Hurtado López ${ }^{1}$
}

\section{Introducción}

Debido al interés que despiertan las ideas de los autores de la teoría del pensamiento complejo para el desarrollo de la investigación científica, especialmente la dedicada a las ciencias de la sociedad, consideramos que el examen de algunos de sus conceptos puede contribuir al esclarecimiento de sus planteamientos; debido a ello es que nos proponemos en este ensayo analizar los conceptos de complejidad y totalidad bajo la perspectiva de Edgar Morin, tal y como aparecen expuestos principalmente en su texto Introducción al pensamiento complejo (Morin, 2008 [1990]). Las tesis expuestas en este ensayo son una aproximación inicial a la obra de este pensador.

En el encuentro de Lisboa, Edgar Morin recibió una serie de observaciones críticas, de cuyo carácter y sentido podemos darnos cuenta por las respuestas que les da el mismo autor (Morin, 2008: 136 ss.). Dichas observaciones se refieren a su "espíritu sintetizador" en relación con su concepción de la complejidad, la "rapidez" de su escritura" y el adjetivo de "confucionista" en el terreno político; y estas críticas no fueron las únicas. En el texto, como queda dicho, no se encuentran publicadas las observaciones, pero él las reconoce todas como inteligentes; sin duda, fueron agudas, puesto que apuntaron a aspectos clave de su obra. Por su parte, Rolando García (2006) dirige su propia crítica a la concepción de Morin respecto de la ciencia clásica y la complejidad, misma que coincide, en parte, con las críticas antes mencionadas, especialmente por su desdén por la obra de Jean Piaget; la relación Morin-Piaget merecería un trabajo aparte, que no es nuestro propósito abordar. De los temas de estas observaciones de García, dada su amplitud, sólo nos ocuparemos en la medida en que nos avoquemos a elaborar las nuestras a algunos conceptos centrales, necesarios para aproximarse al

Fecha de Recepción: 18 de Octubre de 2020. Fecha de Aceptación: 10 de Diciembre de 2020.

1 Profesor en Investigador. Departamento de Economía. CUCEA. Universidad de Guadalajara. Correo electrónico: ahurtado@cucea.udg.mx 
entendimiento de los planteamientos de Edgar Morin. Por lo demás, a lo largo del trabajo y especialmente al final, exponemos nuestras propias observaciones.

\title{
1. El concepto de complejidad. Complejidad y mundo fenoménico
}

El de complejidad es, sin duda, un concepto fundamental. ¿Qué es la complejidad? La pregunta la formula el propio Morin y la responde de la siguiente manera:

\begin{abstract}
A primera vista la complejidad es un tejido (complexus: lo que está tejido en conjunto) de constituyentes heterogéneos inseparablemente asociados: presenta la paradoja de lo uno y lo múltiple. Al mirar con más atención, la complejidad es, efectivamente, el tejido de eventos, acciones, interacciones, retroacciones, determinaciones, azares, que constituyen nuestro mundo fenoménico [todas las cursivas con subrayados son mías: J. H.]. Así es que la complejidad se presenta con los rasgos inquietantes de lo enredado, de lo inextricable, del desorden, la ambigüedad, la incertidumbre... De allí la necesidad, para el conocimiento, de poner orden en los fenómenos rechazando el desorden, de descartar lo incierto, es decir, de seleccionar los elementos de orden y de certidumbre, de quitar ambigüedad, clarificar, distinguir, jerarquizar [...] Pero tales operaciones, necesarias para la inteligibilidad, corren el riesgo de producir ceguera si eliminan a los otros caracteres de lo complejo; y, efectivamente, como ya lo he indicado, nos han vuelto ciegos (Morin, 2008: 32).
\end{abstract}

Antes de comentar este pasaje, permítasenos previamente citar la siguiente definición de complejidad, en donde Morin enfatiza su diferencia respecto al pensamiento "reductor" o "simplificador", pero también del pensamiento "globalista":

Por lo demás, en el segundo volumen de El método, he dicho que la complejidad es la unión de la simplicidad y de la complejidad; es la unión de los procesos de simplificación que implican selección, jerarquización, separación, reducción, con los otros contra-procesos que implican la comunicación, la articulación de aquello que está disociado y distinguido; y es el escapar de la alternativa entre el pensamiento reductor que no ve más que los elementos y el pensamiento globalista que no ve más que el todo (Morin, 2008: 143 y 144).

Debe destacarse el aspecto principal de la definición de complejidad del primer texto, como un conjunto de elementos constitutivos del mundo fenoménico. En efecto, el mundo de las apariencias se presenta como un todo caótico, como una representación caótica del conjunto, cuyas determinaciones y relaciones se le aparecen al observador como un conjunto abigarrado y abstracto (Marx, 1982). El conocimiento occidental cartesiano busca, según Morin, poner orden y certidumbre en esta complejidad que es el mundo complejo, por lo que él lo considera reduccionista. Pero ¿qué es ese orden y certidumbre?, es decir ien qué consiste ese reduccionismo?

Vivimos bajo el imperio de los principios de disyunción, reducción y abstracción, cuyo conjunto constituye lo que llamo el "paradigma de simplificación" (Morin, 2008: 29). 
Pero la complejidad ha vuelto a las ciencias por la misma vía por la que se había ido. El desarrollo mismo de la ciencia física, que se ocupaba de revelar el Orden impecable del mundo, su determinismo absoluto y perfecto, su obediencia a una Ley única [N. B., J. H.] y su constitución de una materia simple primigenia (el átomo), se ha abierto finalmente a la complejidad de lo real. Se ha descubierto en el universo físico un principio hemorrágico de degradación y de desorden (segundo principio de la termodinámica); luego, en el supuesto lugar de la simplicidad física y lógica, se ha descubierto la extrema complejidad microfísica; la partícula no es un ladrillo primario, sino una frontera sobre una complejidad tal vez inconcebible; el cosmos no es una máquina perfecta, sino un proceso en vías de desintegración y, al mismo tiempo, de organización (Morin, 2008: 32 y 33).

\section{Simplificación: desentrañar la esencia detrás de la apariencia}

Deben notarse en este texto las expresiones "revelar un orden del mundo" y "obediencia a una ley única", puesto que constituyen nociones fundamentales. Si la complejidad es el mundo fenoménico y el pensamiento reduccionista o simplificador busca revelar el "orden del mundo", esto es, revelar la ley del fenómeno ("ley única"), expresión ésta de la esencia de las cosas, cuya develación constituye el objeto principal de la labor de la ciencia, entonces iqué es lo que propone el pensamiento complejo? Lo que propone es "sustituir al paradigma de disyunción/reducción/unidimensionalización por un paradigma de distinción/conjunción que permita distinguir sin desarticular, asociar sin identificar o reducir" (Morin, 2008: 34). ¿Pero en qué consiste, a su vez, este último paradigma? Antes de responder a esta pregunta se tiene que determinar aún más lo que caracteriza al "paradigma simplificador". Podría pensarse que la interpretación del pasaje aludido más arriba es forzada, en el sentido de que el paradigma "simplificador" buscaría desentrañar ("reducir") la esencia de las cosas, enunciando la ley del fenómeno, pero aquélla se ve confirmada en el siguiente texto en donde Morin define la complejidad:

Es, efectivamente, el combate con el ángel. Hoy, yo agregaría esto: la complejidad, no es solamente la unión de la complejidad con la no-complejidad (la simplificación); la complejidad se halla en el corazón de la relación entre lo simple y lo complejo porque una relación tal es, a la vez, antagonista y complementaria.

Creo profundamente que el mito de la simplicidad ha sido extraordinariamente fecundo para el conocimiento científico que quiere ser un conocimiento no trivial, que no busca a nivel de la espuma de los fenómenos, sino que busca lo invisible detrás del fenómeno. Bachelard decía: "No hay otra ciencia que la de lo oculto". Pero, buscando lo invisible, encontramos, detrás del mundo de las apariencias //145/ y de los fenómenos, el tras-mundo de las leyes que, en conjunto, constituyen el orden del mundo [J. H.]. Si seguimos este proceso, llegamos a la visión de un tras-mundo más real que el mundo real porque está fundado sobre el orden, y nuestro mundo real tiende a devenir un poco, como en la filosofía hinduista, el mundo de las apariencias [Nota Bene, J. H.] de maya, de las ilusiones, de los epifenómenos (Morin, 2008: 144 y 145). 
El verdadero problema, al que volveré, es que ese mundo de las apariencias, de los epifenómenos, del desorden, de las interacciones es, al mismo tiempo, nuestro mundo, y que, en el tras-mundo, no existe el orden soberano, sino otra cosa (Morin, 2008: 145).

Así pues, el pensamiento científico "simplificador" se propone desentrañar el orden del mundo, la ley del fenómeno, la esencia detrás de la apariencia; este "mito de la simplicidad" reconoce Morin que ha sido "extraordinariamente fecundo para el conocimiento científico", pero su razonamiento consiste en que, como el mundo de las apariencias es nuestro mundo, detrás de ellas no existe "otra cosa", es decir, no existe la esencia, sea ésta compleja o simple:

Nuestro universo, en el cual todas las cosas están separadas en y por el espacio es, al mismo tiempo, un universo en el que no hay separación. Esto muestra que, en nuestro universo de la distinción, hay una otra cosa (¿por detrás?) en la cual no hay distinción. En el plano de la complejidad, eso quiere decir que en el tras-mundo no hay ni complejidad, ni simplicidad, ni orden, ni desorden, ni organización. Ahora bien, algunos podrían reconsiderar, desde este ángulo, las ideas taoístas sobre el vacío insondable considerado como realidad única y fundamental (Morin, 2008: 145).

El plano de la complejidad es el mundo fenoménico, el plano de la representación caótica del conjunto, pero en el tras-mundo, es decir, detrás de las apariencias, dice Morin, no existe nada. El resultado de todo esto, pues, es que el pensamiento complejo rechaza la idea misma de la esencia, sea ésta compleja o simple, la esencia misma "es inconcebible": No obstante, la complejidad es definida como la contradicción ("la dialógica") ¿entre qué? entre el orden y el desorden, entre la esencia "inconcebible" (i) y la apariencia. Pero detrás de la complejidad, es decir, en la contradicción, las distinciones, esto es, la contradicción misma no se niega/asume, es decir, no se supera (aufheben), sino que "se esfuma". A la "reducción" del fenómeno a su ley por la ciencia clásica, opone entonces el pensamiento complejo, entendido de esta manera. Así dice:

Para mí, la idea fundamental de la complejidad no es que la esencia del mundo es compleja y no simple. Es que esa esencia es inconcebible [N. B., J. H.]. La complejidad es la dialógica orden/desorden/organización. Pero, detrás de la complejidad //146/, el orden y el desorden se disuelven, las distinciones se esfuman [N. B., J. H.]. El mérito de la complejidad es el de denunciar la metafísica del orden. Como lo dijera muy bien Whitehead, detrás de la idea de orden hay dos cosas: la idea mágica de Pitágoras de que los números son la realidad última, y la idea religiosa todavía presente, tanto en Descartes como en Newton, de que el entendimiento divino es el fundamento del orden del mundo. Ahora bien, cuando uno ha retirado al entendimiento divino y a la magia de los números, ¿qué queda? ¿Las leyes? ¿Una mecánica cósmica autosuficiente? ¿Es la realidad verdadera? ¿Es la naturaleza verdadera? A esa visión débil, yo opongo la idea de la complejidad (Morin, 2008: 145 y 146).

Al margen de cuál es la concepción de Descartes y de Newton respecto a la cuestión (el cual es un tema aparte), aquí el punto en discusión es la propia concepción de Mo- 
rin acerca de su concepto de realidad. Pero precisamente en relación con las ideas de Morin expuestas en su obra El método (Morin, 1983 y 1986), y aquí reiteradas, acerca de la ciencia clásica, dice Rolando García:

El gran prestigio de Morin en su propio campo no parece transferible a otros dominios. Las afirmaciones que hemos citado [de El método] bordean una posición oscurantista y no se justifican frente al desarrollo histórico de la ciencia. En primer lugar, no hubo una tal "ruina de la física clásica". En segundo lugar, "la complejidad de la física nueva" no se caracteriza por "el oscurecimiento, desorden, incertidumbre y antinomia". Dicho de otra manera, ni la física de Newton está en ruinas, puesto que se sigue aplicando para lanzar un misil que destruya una casa de un supuesto terrorista, ni "la física nueva" (suponiendo que Morin se refiera a la relatividad y a la mecánica cuántica) "emergió como oscurecimiento y desorden" (García, 2006: 209).

En el mismo tenor, le hubieron de dirigir críticas en el mencionado encuentro de Lisboa, puesto que tuvo que reconocer la complejidad de hecho de la ciencia moderna (cf. Morin, 2008: 147 y 148).

No puede quedar duda, entonces, de la exactitud de nuestra interpretación, expuesta más arriba, puesto que Morin reitera la idea de que el pensamiento "complejo", en oposición al pensamiento "simplificador", no se propone revelar la "esencia del mundo":

Dentro de ese marco, yo diría que acepto plenamente relativizar la complejidad. Por una parte, ella integra a la simplicidad y, por otra parte, se abre sobre lo inconcebible. Estoy totalmente de acuerdo con esas condiciones para aceptar la complejidad como principio del pensamiento que considera al mundo, y no como el principio revelador de la esencia del mundo (Morin, 2008: 146).

La complejidad no es un fundamento, es el principio regulador que no pierde nunca de vista la realidad del tejido fenoménico en la cual estamos y que constituye nuestro mundo (Morin, 2008: 146).

\section{La esencia y la apariencia}

Naturalmente, no se puede negar la importancia de exponer las categorías y relaciones del mundo fenoménico. Pero el mundo de las apariencias es incomprensible si no se comprende la ley del fenómeno, es decir la esencia que se aparece. De otra manera no se comprende ni lo uno ni lo otro. Claro está que la ciencia no sólo debe proponerse encontrar las determinaciones esenciales, sino exponer también las formas de aparición. La realidad efectiva constituye el contenido de la ciencia, y de la realidad efectiva debe saberse que:

Wirklichkeit [realidad efectiva] no es una realidad cualquiera, sino una realidad cualificada como unidad de fenómeno y esencia. En otras palabras, se trata de una realidad fenoménica que no es mero fenómeno o apariencia, sino una realidad que por adecuarse al concepto 
es verdadera, plenamente racional y eficaz o efectiva (Hegel, 1999, nota 86 de Valls Plana al 6 , p. 105).

Rechazar el concepto de esencia es rechazar la relación interna entre fenómeno y esencia. Es establecer un báratro infranqueable entre fenómeno y esencia y es también negar el concepto de contradicción como una determinación de la reflexión, es decir, esencialidad, en la que la esencia es puesta como apariencia, reflexión.

\section{El concepto de totalidad}

Para Morin la totalidad es una "unidad compleja" (2008: 81), pero debe diferenciarse de la "completud" (Morin, 2008: 100), puesto que si bien es verdad que "no podemos aislar los objetos unos de otros. En última instancia, todo es solidario. Si tenemos sentido de la complejidad, tenemos sentido de la solidaridad. Más aún, tenemos sentido del carácter multidimensional de toda realidad” (Morin, 2008: 100) La visión compleja de las ciencias sociales implica pensar cada realidad conteniendo a las otras dimensiones, es decir, de manera multidimensional, de otra manera el aislar una esfera de la otra es parcial, pobre, unidimensional (Morin, 2008: 100). Es necesario que cada dimensión sea relacionada con las otras; "de allí la creencia de que podemos identificar la complejidad con la completud" (Morin, 2008: 100):

En un sentido, yo diría que la aspiración a la complejidad lleva en sí misma la aspiración a la completud, porque sabemos que todo es solidario y multidimensional. Pero, en otro sentido, la conciencia de la complejidad nos hace comprender que no podremos escapar jamás a la incertidumbre y que jamás podremos tener un saber total: "la totalidad es la no verdad" (Morin, 2008: 100 y 101).

Esta última cita de Adorno es matizada por Morin; sin embargo, en el sentido de que la totalidad es, a la vez, la verdad y la no verdad, puesto que "la aspiración a la totalidad es una aspiración a la verdad y que el reconocimiento de la imposibilidad de la totalidad es una verdad muy importante" (Morin, 2008: 137).

Respecto a la categoría de totalidad concreta, por supuesto que la totalidad no es todos los hechos, sino la realidad como un todo estructurado y dialéctico (Kosík, 1983: 55). El concreto real se encuentra fuera de nosotros, pero en el mundo pensado se parte de una representación caótica del conjunto, es el concreto representado. El proceso analítico de la investigación científica arriba a determinaciones abstractas. Éste es el camino de lo concreto a lo abstracto. El camino de regreso es de lo abstracto a lo concreto, mediante el cual se reproduce por la vía del pensamiento ese concreto, pero como concreto pensado, como totalidad orgánica.

El conocimiento de los hechos no es posible como conocimiento de la realidad más que en ese contexto que articula los hechos individuales de la vida social en una totalidad como momentos del desarrollo social. Este conocimiento parte de las determinaciones naturales, inmediatas, puras, simples (en el mundo capitalista), recién caracterizadas, para avanzar 
desde ellas hasta el conocimiento de la totalidad concreta como reproducción intelectual de la realidad (Lukács, 1969: 10).

Esta consideración dialéctica de la totalidad, que tanto se aleja, aparentemente, de la realidad inmediata que la realidad parece tan "acientíficamente" construida, es verdaderamente el único método que permite reproducir y captar intelectualmente la realidad. La totalidad concreta es, pues, la categoría propiamente dicha de la realidad [realidad efectiva] (Lukács, 1969: 11).

La categoría de realidad responde a la pregunta más general de ¿qué es la realidad? La realidad es una totalidad articulada que está creándose. Pero el concreto real, lo empírico morfológico, es el mundo de la pseudoconcreción, el fundo de las apariencias, en donde las cosas aparecen invertidas. Mediante el proceso de la investigación científica debe irse más allá de esa apariencia y desentrañar la esencia de las cosas, pero además exponer la razón de ser de las formas de aparición y éstas mismas.

El mundo de la pseudoconcreción es un claroscuro de verdad y engaño. Su elemento propio es el doble sentido. El fenómeno muestra la esencia y, al mismo tiempo, la oculta. La esencia se manifiesta en el fenómeno, pero sólo de manera inadecuada, parcialmente, en algunas de sus facetas y ciertos aspectos. El fenómeno indica algo que no es él mismo, y existe gracias a su contrario. La esencia no se da inmediatamente; es mediatizada por el fenómeno y se muestra, por tanto, en algo distinto de lo que es. La esencia se manifiesta en el fenómeno. Su manifestación en éste revela su movimiento y demuestra que la esencia no es inerte y pasiva. Pero, igualmente, el fenómeno revela la esencia. La manifestación de la esencia es la actividad del fenómeno (Kosík, 1983: 27).

Pero en todo momento debe asumirse que la esencia determina la apariencia:

[...] la esencia es la verdad del ser en tanto ser que ha ido adentro de sí o ser que está-siendo dentro de sí; la distinción de la esencia respecto del ser inmediato la constituye esa reflexión, su parecer [o brillar] dentro de sí, y ésta [la reflexión] [y, ulteriormente, el fenómeno, J. H.] es la determinación propia de la esencia (Hegel, 1999: 211).

La apariencia, es el aparecer de la esencia misma. La apariencia es determinación de la esencia. Suprimir la esencia y atenerse al puro fenómeno es un abstracción violenta, puramente unilateral, puesto que no existe aparición sin esencia que haya que aparecer y, por otra parte, la esencia no aparece desnuda, sino como apariencia o fenómeno.

En virtud de que la esencia — a diferencia de los fenómenos- no se manifiesta directamente, y por cuanto que el fundamento oculto de las cosas debe ser descubierto mediante una actividad especial, existen la ciencia y la filosofía. Si la apariencia fenoménica y la esencia de las cosas coincidieran totalmente, la ciencia y la filosofía serían superfluas (Kosík, 1983: 29). 


\section{La aplicación del método de la complejidad al análisis de los movimientos sociales}

La teorización sobre la complejidad desarrollada en el texto de la Introducción es sumamente abstracta. De ahí que su aplicación a la investigación concreta plantee dificultades al investigador. Por eso, la indicación que hace Morin en relación con la aplicación concreta del método de la complejidad en sus trabajos que involucran un análisis empírico, particularmente el de los acontecimientos de 1968 en París, constituyen un referente de interés para ver en acción el mencionado método. Éste es contrapuesto al método de otros "científicos" pretenciosos, dice Morin, a quienes, hay que decirlo, no identifica expresamente.

El único ideal era el de aislar las variables en juego en las interacciones permanentes en un sistema, pero nunca el de considerar con precisión las interacciones permanentes del sistema. Así, paradójicamente, los estudios ingenuos, en la superficie de los fenómenos, eran mucho más complejos, es decir, en última instancia, "científicos", que los pretenciosos estudios cuantitativos sobre estadísticas inmensas, guiadas por pilotos de poco cerebro. Así lo eran, digo con falta de modestia, mis estudios fenoménicos que intentaban aprehender la complejidad de una transformación social multidimensional en una comunidad de Bretaña o, los estudios en vivo del florecimiento de los acontecimientos de mayo del 68. Yo no tenía por método nada más que tratar de aclarar los múltiples aspectos de los fenómenos, e intentar aprehender las relaciones cambiantes. Relacionar, relacionar siempre, era un método más rico, incluso a nivel teórico, que las teorías blindadas, guarnecidas epistemológica y lógicamente, metodológicamente aptas para afrontar lo que fuere salvo, evidentemente, la complejidad de lo real (Morin, Lefort y Castoriadis, 2009: 59, nota 7).

Debe notarse que Morin caracteriza sus estudios, no sin ironía, como "ingenuos", mismos que se realizan en "la superficie de los fenómenos", los cuales considera más complejos que aquellos hechos sobre "estadísticas inmensas". Su método lo define como "nada más que tratar de aclarar los múltiples aspectos de los fenómenos, e intentar aprehender las relaciones cambiantes", lo que está en todo congruente con su definición de complejidad, pues se mantiene en el plano del mundo fenoménico.

La crítica a Morin de Rolando García se refiere en este contexto, a su dificultad, en el plano metodológico, de la aplicación de su análisis de la complejidad:

Sin embargo, su crítica no ofrece una formulación precisa de los problemas que enuncia (problemas que el cartesianismo dejó pendientes y que corresponden al campo de la teoría del conocimiento) como para conducir a una metodología de trabajo aplicable a las situaciones concretas que él considera como "complejas" (García, 2006: 21).

Es en este plano en el que se debe profundizar más en el estudio de la propuesta teórica de Morin, puesto que su contribución al análisis social es un aspecto de especial interés. 


\section{Algunas observaciones finales}

El estilo expositivo de Morin es abstracto en el sentido de no deducir sus categorías, sino sólo aseverarlas o enunciarlas. Deja muchas puertas abiertas a la indeterminación, en un afán pretendidamente "abierto" en la teorización. Es declamación en prosa. Las referencias a quienes se refiere son oscuras, quedan en la indeterminación. Cuando es lo suficientemente preciso en sus referencias, sus interpretaciones son cuestionables (véanse las observaciones de Rolando García respecto a la ciencia clásica) y no necesariamente por una supuesta heterodoxia. Existe ambigüedad y confusión en la formulación de sus conceptos. Sus expresiones son paradojales, y no siempre necesaria o genuinamente (auténticamente) dialécticas. Su pensamiento es confuso puesto que, no obstante reivindicar la contradicción, se mantiene en el ámbito del mundo fenoménico y se establece en él o lo ensalza.

En relación con la dialéctica de Hegel como idea de la complejidad, es una exageración de Morin identificar los males que le atribuye al paradigma "simplificador", con el pensamiento occidental como un todo. Podría denominarse francocentrismo el "reducir" el pensamiento occidental a la cultura filosófica francesa (cartesianismo y clericalismo). Esto lo reconoce al decir que en la dialéctica de Hegel, indiscutible representante del pensamiento occidental, está formulada la "idea de la complejidad".

La idea de complejidad estaba mucho más diseminada en el vocabulario común que en el científico. Llevaba siempre una connotación de advertencia al entendimiento, una puesta en guardia contra la clarificación, la simplificación, la reducción demasiado rápida. De hecho, la complejidad tenía también delimitado su terreno, pero sin la palabra misma, en la filosofía: en un sentido, la dialéctica, y en el terreno lógico, la dialéctica hegeliana, eran su dominio, porque esa dialéctica introducía la contradicción y la transformación en el corazón de la identidad (Morin, 2008: 58).

Rinde tributo, además, a la filosofía de Hegel y no niega las imputaciones de hegelianismo, atribuyéndose una filiación al mismo:

He leído un texto que decía que hay un hegelianismo disimulado [N. B., J. H.] en mis concepciones. Mi concepción en relación con ello, es a la vez, compleja y clara. Lo que me fascina en Hegel, es la confrontación de las contradicciones que se presentan sin cesar al espíritu, y es el reconocimiento del rol de la negatividad. No es la síntesis, el Estado absoluto, el Espíritu absoluto (Morin, 2008: 137).

Sin embargo, si bien Morin emplea y discute las categorías de la ontología y la lógica expuestas en la filosofía de Hegel: tales como las de totalidad, causalidad, sujeto, objeto, devenir, identidad, contradicción, simple, complejo, etc., etc., debe decirse que expone su peculiar y propia concepción de ellas. Decir, por otra parte, que la complejidad tenía delimitado su terreno en la filosofía pero sin la palabra o término, es un desatino pues iqué es la dialéctica de Hegel, sino un sistema de la ciencia, de la "complejidad", un círculo de círculos? Los tres principios que expone en su texto, son en su esencia tomados de Hegel: 1) el dialógico, es la relación de causalidad; 2) el 
recursivo, es la acción recíproca, y 3) el hologramático, es tomado de la lógica de la reflexión ( $c f$. Morin, 2008: 105 ss.). Podría parecer que Morin defiende la dialéctica y la "idea" de contradicción como puntal de su reflexión, pero la fuerza, la potencia de la contradicción se "esfuma" en sus manos. Pero hay que exculpar a Morin de la acusación de hegelianismo (por lo menos en los conceptos discutidos en este texto). La suya no es una dialéctica genuina, puesto que al negar la esencia y, por lo tanto, en el poner de la esencia, la génesis de la contradicción, niega el motor de la dialéctica. No comprende la relación entre esencia y apariencia. La dialéctica, despojada de bases materialistas, es bien vista por los intelectuales de la burguesía [y el mundo académico oficial]. La dialéctica, como un juego artificioso de conceptos es aceptable en el campo de la concepción burguesa de la sociedad y del mundo. La dialéctica materialista es vista como "arrogante" y "reduccionista". Si bien se afirma que la contradicción es una idea central de su discurso. Así pues, si bien Morin reivindica el concepto de contradicción, niega la esencia, la estructura íntima de la realidad, y se mantiene, en la investigación de la complejidad, en el mundo fenoménico, en el terreno de la inversión y de la realidad fetichizada.

No obstante, debe decirse que al considerar el contexto del predominio del pensamiento positivista, la propuesta teórica de Morin es una contribución a la reflexión y al esfuerzo de comprensión del desarrollo de la ciencia actual. Su reivindicación de la dialéctica y de la idea de contradicción como uno de sus ejes de razonamiento es bienvenida y es estimulante, dado el páramo en el que aparece el pensamiento dominante.

\section{Referencias bibliográficas}

García, R. (2006). Sistemas complejos, conceptos, método y fundamentación epistemológica de la investigación interdisciplinaria. Buenos Aires: Gedisa.

Hegel, G. W. F. (1999). Enciclopedia de las ciencias filosóficas en compendio (trad. Ramón Valls Plana). Madrid: Alianza Editorial.

Kosík, K. (1983). Dialéctica de lo concreto (trad. Adolfo Sánchez Vázquez, 9ạ edición). México: Grijalbo.

Lukács, G. (1969). Historia y conciencia de clase (trad. Manuel Sacristán). México: Grijalbo.

Marx, K. (1982). Introducción general a la crítica de la economía política/1857 (trad. José Aricó y Jorge Tula, 15a edición). México: Cuadernos de Pasado y Presente, núm. 1.

Morin, Edgar. (1983). El método. 2. La vida de la vida (trad. Ana Sánchez). Madrid: Cátedra.

—_. (2006 [1986]). El método. 3. El conocimiento del conocimiento (trad. Ana Sánchez, 5aㅡ edición). Madrid: Cátedra.

—. (2008 [1990]). Introducción al pensamiento complejo. Buenos Aires: Gedisa. (Barcelona 1995).

Morin, Edgar, Lefort, Claude, y Castoriadis, Cornelius. (2009). Mayo del 68: La brecha (trad. de Ricardo Aguilera). Buenos Aires: Nueva Visión. 